\title{
Special Considerations for Elderly Patients with Head and Neck Cancer during the COVID-19 Pandemic
}

\author{
Arun Sharma, MD, MS, FACS ${ }^{1}$ \\ ${ }^{1}$ Southern Illinois University School of Medicine
}

April 28, 2020

\begin{abstract}
Elderly head and neck cancer patients are at increased risk of adverse outcomes during and after treatment of head and neck cancer. COVID-19 severity and mortality can be expected to be significantly greater in elderly head and neck cancer patients, given that increased age, comorbidities, and presence of malignancy are known risk factors for disease severity and mortality in COVID-19 patients. Therefore, their management requires multidisciplinary consensus and patient input. A thorough geriatric assessment, which has been shown to be beneficial prior to the COVID-19 pandemic, could be particularly helpful in this patient population with the added dimension of COVID-19 risk. In many cases, prudent treatment plan modification may allow for overall best outcomes. Furthermore, recruitment of social services and, when appropriate, palliative care, may allow for optimal management of these patients.
\end{abstract}

Arun Sharma, MD, MS ${ }^{1}$, Dana L. Crosby, MD, MPH ${ }^{1}$

${ }^{1}$ Department of Otolaryngology - Head and Neck Surgery, Southern Illinois University School of Medicine, Springfield, Illinois

Grant support: None

This work has not been presented at any meetings.

Brief running title: Elderly head and neck cancer patients and COVID-19

Key words: COVID-19; SARS-CoV-2; novel coronavirus; head and neck cancer; geriatrics

\section{Corresponding Author:}

Arun Sharma, MD, MS, FACS

Associate Professor

Department of Otolaryngology-Head and Neck Surgery

Southern Illinois University School of Medicine

720 N Bond Street

Springfield, Illinois 62794

Email: asharma74@siumed.edu

${ }^{*}$ Special consideration for Elderly Patient with Head and Neck Cancer during the COVID-19 Pandemic

Abstract 
Elderly head and neck cancer patients are at increased risk of adverse outcomes during and after treatment of head and neck cancer. COVID-19 severity and mortality can be expected to be significantly greater in elderly head and neck cancer patients, given that increased age, comorbidities, and presence of malignancy are known risk factors for disease severity and mortality in COVID-19 patients. Therefore, their management requires multidisciplinary consensus and patient input. A thorough geriatric assessment, which has been shown to be beneficial prior to the COVID-19 pandemic, could be particularly helpful in this patient population with the added dimension of COVID-19 risk. In many cases, prudent treatment plan modification may allow for overall best outcomes. Furthermore, recruitment of social services and, when appropriate, palliative care, may allow for optimal management of these patients.

Coronavirus disease 2019 (COVID-19), caused by severe acute respiratory syndrome coronavirus 2 (SARS$\mathrm{CoV}-2)$, has been designated as a pandemic by the World Health Organization (WHO).(1, 2) There remain many uncertainties about the disease and its optimal management;(3) even greater uncertainties are present in management of diseases not directly related to COVID-19 during the pandemic. Since initial reports suggest high viral load in the pharynx and respiratory secretions, there are important ramifications for optimal management of patients with head and neck cancer. This is especially true of patients with mucosal malignancies and those who require tracheotomy or laryngectomy.(4-6) As is standard for management of head and neck cancers, multidisciplinary tumor board and conferences are critical for ensuring the safety of patients, caregivers, family members, healthcare providers, and the greater community.(6-8) Elderly patients with head and neck cancer are a unique subset as they are at increased risks of adverse outcomes; $70 \%$ of deaths from head and neck cancer occur in patients over the age of 70.(9) Therefore, their management deserves close attention, multidisciplinary consensus, and shared decision making.

The National Institute on Aging and US Census Bureau define elderly patients as those over the age of 65.(10) However, much of the oncology literature uses cutoffs ranging from $70-80$ for classifying patients as elderly or geriatric. $(9,11)$ Regardless of the age cutoff to define "elderliness", age is best thought of a continuous variable, with increased risks occurring as a continuum rather than after a certain chronological age. Furthermore, the impacts of age on survival and functional outcomes are modified by comorbidities, frailty, and functional status. (9, 12-14) Increasing age, presence of malignancy, and comorbidity are associated with increased disease severity in COVID-19,(1, 15-17) which puts elderly head and neck cancer patients at risk of poor outcomes from both diseases.

Treatment of head and neck cancer can involve surgery, radiation therapy, systemic therapy, or a combination of these. Advanced cancers generally require multimodality therapy. Whether and how the current COVID19 pandemic should modify treatment modalities is currently unclear, especially for elderly patients.(6) However, COVID-19 patients who are 80 years old or older have a mortality risk of 13\%.(18) Furthermore, $95 \%$ of deaths from COVID-19 occur in patients over $60 ; 50 \%$ of deaths occur in patients over 80.(19) Given concerns about hospital-acquired COVID-19,(17) surgery should certainly be reconsidered for elderly patients as it can result in exposure to COVID-19, which is particularly concerning given the risk of disease severity and mortality. However, administration of both radiation therapy and systemic therapy require repeated visits, although inpatient settings can sometimes be avoided. Therefore, until more robust data becomes available, treatment decisions will need to be individualized and take into account patient disease and status, local COVID-19 burden, and resource availability.

The International Society of Geriatric Oncology consensus is to recommend a geriatric assessment (GA) in older patients with cancer.(11) GA includes assessment of functional status, comorbidity, cognition, mental health status, fatigue, social status and support, nutrition, and presence of geriatric syndromes. The benefits of such an assessment include more accurate prediction of adverse outcomes, detection of unidentified problems, improved estimation of residual life expectancy, appropriate employment of geriatric interventions, and appropriate cancer treatment selection.(11) The potential impact of COVID-19 infection could be assessed within this GA framework to allow for optimal shared decision making. Therefore, an 85 year old head and neck cancer patient with multiple comorbidities and limited social support may be best served by delaying or altering the treatment plan given the potential harm that could occur with COVID-19 infection. 
However, a 70 year old patient with a similar cancer but no comorbidities and strong social support may be able to proceed with treatment, with appropriate precautions for both the patient and healthcare providers. Certainly, such decisions would need to be made after multidisciplinary discussion, extensive counseling, and shared decision making.

Early involvement of comprehensive palliative care and social services are often an integral part of management of elderly head and neck cancer patients, especially in the setting of recurrent or metastatic disease.(13) Such services are likely to be particularly helpful during the current COVID-19 pandemic.

The current COVID-19 pandemic is altering how we manage head and neck cancer in a multitude of ways. This is particularly evident in elderly head and neck cancer patients. This particularly vulnerable population is even more susceptible to adverse outcomes during the COVID-19 pandemic. For those reasons, we have a responsibility to provide multidisciplinary care, thorough assessment of risks and benefits of any possible interventions, shared decision making, social resources, and, when appropriate, comprehensive palliative care for elderly head and neck cancer patients.

References

1. Guan WJ, Liang WH, Zhao Y, Liang HR, Chen ZS, Li YM, et al. Comorbidity and its impact on 1590 patients with Covid-19 in China: A Nationwide Analysis. Eur Respir J. 2020 Mar 26.

2. World Health Organization. Coronavirus (COVID-19) events as they happen. [cited 2020 4/8/2020]; Available from: https://www.who.int/emergencies/diseases/novel-coronavirus-2019/events-as-they-happen.

3. Poston JT, Patel BK, Davis AM. Management of Critically Ill Adults With COVID-19. JAMA. 2020.

4. David A, Russell M, El-Sayed I, Russell M. Tracheostomy guidelines developed at a large academic medical center during the COVID-19 pandemic. Epub 4/9/20. Head \& Neck.

5. Tay JK, Khoo ML, Loh WS. Surgical Considerations for Tracheostomy During the COVID-19 Pandemic: Lessons Learned From the Severe Acute Respiratory Syndrome Outbreak. JAMA Otolaryngol Head Neck Surg. 2020 Mar 31.

6. Crosby D, Sharma A. Evidence-Based Guidelines for Management of Head and Neck Mucosal Malignancies during the COVID-19 Pandemic. Manuscript currently under review.

7. Lewis CM, Nurgalieva Z, Sturgis EM, Lai SY, Weber RS. Improving patient outcomes through multidisciplinary treatment planning conference. Head \& neck. 2016 Apr;38 Suppl 1:E1820-5.

8. Liu JC, Kaplon A, Blackman E, Miyamoto C, Savior D, Ragin C. The impact of the multidisciplinary tumor board on head and neck cancer outcomes. The Laryngoscope. 2020 Apr;130(4):946-50.

9. Cervenka BP, Rao S, Bewley AF. Head and Neck Cancer and the Elderly Patient. Otolaryngologic clinics of North America. 2018 Aug;51(4):741-51.

10. US Census Bureau. SIXTY-FIVE PLUS IN THE UNITED STATES. [4/12/20]; Available from: https://www.census.gov/population/socdemo/statbriefs/agebrief.html.

11. Wildiers H, Heeren P, Puts M, Topinkova E, Janssen-Heijnen MLG, Extermann M, et al. International Society of Geriatric Oncology Consensus on Geriatric Assessment in Older Patients With Cancer. Journal of Clinical Oncology. 2014;32(24):2595-603.

12. Noor A, Gibb C, Boase S, Hodge JC, Krishnan S, Foreman A. Frailty in geriatric head and neck cancer: A contemporary review. The Laryngoscope. 2018 Dec;128(12):E416-e24.

13. Mady LJ, Nilsen ML, Johnson JT. Head and Neck Cancer in the Elderly: Frailty, Shared Decisions, and Avoidance of Low Value Care. Clinics in geriatric medicine. 2018 May;34(2):233-44.

14. Genther DJ, Gourin CG. Effect of comorbidity on short-term outcomes and cost of care after head and neck cancer surgery in the elderly. Head \& neck. 2015 May;37(5):685-93. 
15. Weiss P, Murdoch DR. Clinical course and mortality risk of severe COVID-19. The Lancet. 2020;395(10229):10145 .

16. Guan W-j, Ni Z-y, Hu Y, Liang W-h, Ou C-q, He J-x, et al. Clinical Characteristics of Coronavirus Disease 2019 in China. New England Journal of Medicine. 2020.

17. Wang D, Hu B, Hu C, Zhu F, Liu X, Zhang J, et al. Clinical Characteristics of 138 Hospitalized Patients With 2019 Novel Coronavirus-Infected Pneumonia in Wuhan, China. JAMA. 2020;323(11):1061-9.

18. Verity R, Okell LC, Dorigatti I, Winskill P, Whittaker C, Imai N, et al. Estimates of the severity of coronavirus disease 2019: a model-based analysis. The Lancet Infectious Diseases.

19. World Health Organization. Statement - Older people are at highest risk from COVID-19, but all must act to prevent community spread. [4/13/20]; Available from: http://www.euro.who.int/en/healthtopics/health-emergencies/coronavirus-covid-19/statements/statement-older-people-are-at-highest-risk-fromcovid-19,-but-all-must-act-to-prevent-community-spread. 